EPJ Web of Conferences 60, 20026 (2013)

DOI: $10.1051 /$ epjconf $/ 20136020026$

C Owned by the authors, published by EDP Sciences, 2013

\title{
Search for the SM Higgs boson decaying to bb in associated production with a $\mathrm{Z}$ boson decaying in the invisible channel
}

\author{
Silvio Donato ${ }^{1,2, a}$ \\ ${ }^{1}$ Scuola Normale Superiore and INFN, Pisa, Italy
}

\begin{abstract}
A search for the Standard Model (SM) Higgs boson decaying into two b jets using associated production with a $\mathrm{Z}$ boson decaying into a pair of neutrinos is presented at LHCP. The CMS pp collisions data-samples of $4.7 / \mathrm{fb}$ of at the center-of-mass energy of $7 \mathrm{TeV}$ and $19.0 / \mathrm{fb}$ at the energy of $8 \mathrm{TeV}$ have been analyzed. The techniques employed to discriminate signal from background are explained. An upper limit of 2.3 times the SM Higgs cross section at $95 \%$ of confidence level has been observed. The signal strength for $m_{H}=125 \mathrm{GeV}$ is $1.0 \pm 0.8$ times the $\mathrm{SM}$ prediction.
\end{abstract}

\section{Introduction}

The CMS and ATLAS collaborations have announced the discovery of a new particle [1,2] compatible with the Standard Model (SM) Higgs boson [3, 4]. The new particle has been observed mainly in the $\gamma \gamma, Z Z$ and $W W$ decay channels and its mass is about $m_{H}=125 \mathrm{GeV}$. At this mass, the Standard Model predicts that the branching ratio of the Higgs boson decay in a pair of $b$ jets is about $58 \%$. The observation of this decay channel is important to measure the coupling of the new particle to fermions, and particularly to quarks.

The huge amount of QCD background makes impossible to trigger only on the bb final state in the direct production $p p \rightarrow H \rightarrow b \bar{b}$. To reject the QCD background, we exploit the associated production of the Higgs boson with the vector boson $W$ or $Z$, that is quite large due to the large coupling of the Higgs boson to the vector bosons. This paper describes the search of the Higgs boson in $p p \rightarrow Z(v \bar{v}) H(b \bar{b})$ channel performed by the CMS collaboration.

A detailed description of CMS detector [5] and $W / Z(l v / v \bar{v} / l l) H(b \bar{b})$ analysis [6] can be found elsewhere.

\section{Signal topology}

The signal events are characterized by the presence of two high $p_{T} \mathrm{~b}$ jets, from the Higgs boson decay, and a large missing transverse energy (MET), from $\mathrm{Z}$ decay. The MET and the Higgs boson candidate are supposed to be back-to-back. Additional light jets can arise from final and initial state radiation, pile-up and underlying event. The signal topology does not produce isolated leptons. However, some non-isolated leptons can be produced by $\mathrm{B}$ hadrons decays. The main backgrounds to this channel are: $t \bar{t}, Z / W+$ jets and $Z Z / Z W / W W$.

\footnotetext{
ae-mail: silvio.donato@cern.ch
}
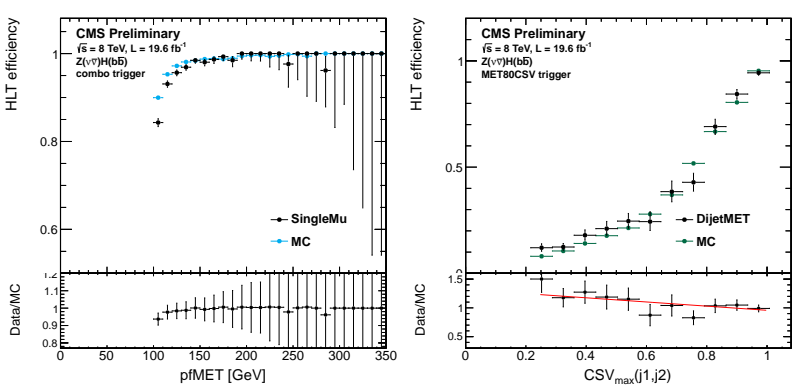

Figure 1. Efficiency of the combined trigger as a function of the offline MET in $t \bar{t}$ control region (left). Efficiency of the btagging trigger path as function of the best b-tagged jet discriminator (right).

\section{Trigger}

In order to trigger signal-like data, three variables have been used: MET, jet $p_{T}$ and jet b-tag discriminator (CSV [7]). The hardware trigger (Level-1) selects events with MET > $36-40 \mathrm{GeV}$ (depending on luminosity). The high level trigger (HLT) accepts events that have passed at least one of the following requirements:

- $\mathrm{MET}>150 \mathrm{GeV}$;

- MET > $100 \mathrm{GeV}$, two central jets $(|\eta|<2.6)$ with $p_{T}$ above 60 and $25 \mathrm{GeV}$, a modulus of the vectorial sum larger then $100 \mathrm{GeV}$ and no jets with $p_{T}>40 \mathrm{GeV}$ and $\Delta \phi($ jet, MET $)<0.5$;

- $\mathrm{MET}>80 \mathrm{GeV}$, two central jet with $p_{T}>30 \mathrm{GeV}$ and one b-tagged jet.

The left side of the Figure 1 shows the combined trigger efficiency as a function of the offline MET as measured on single muon triggered events in the $t \bar{t}$ enriched region for data and simulations. The efficiency has a plateau near 


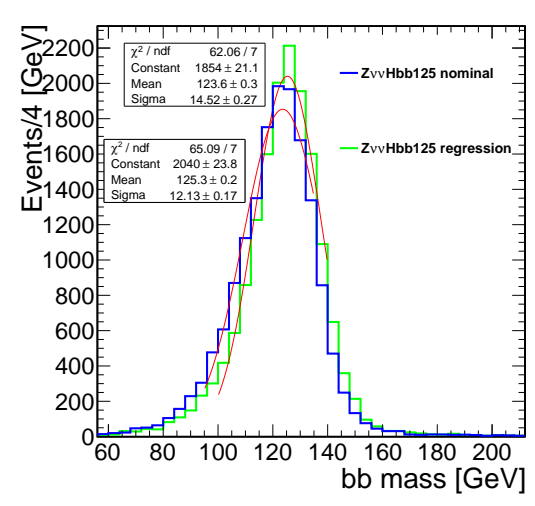

Figure 2. Invariant mass of the b-jet pair in simulated signal event before (blue) and after (green) applying the regression. The resolution improves by about $15 \%$.

$100 \%$ starting from MET $>170 \mathrm{GeV}$. The efficiency is well reproduced by the simulations. Small discrepancies appearing in the low MET region have been corrected applying scale factors. The differences among scale factors obtained in different control regions have been taken in account as systematic uncertainties.

The right side of the Figure 1 plots the b-tagging trigger efficiency as a function of the best b-tagged jet discriminator. It has been measured in a sample triggered by di-jet plus MET. As for MET trigger efficiency, scale factors have been obtained in order to correct the simulated trigger efficiency.

\section{Jet energy regression}

In attempt to get the true b-jet energy, a Boost Decision Tree (BDT) has been trained based on variables potentially sensitive to an energy mis-measurement:

- calorimeter energy deposit;

- jet constituents;

- features of the tracks (number of hits, $p_{T}$ and impact parameter);

- properties of the secondary vertex (when it exist);

- variables of the jet soft leptons (if they are).

Using the correction computed with the BDT, the new energy value is closer to the real quark energy. Figure 2 shows the invariant mass of the two jets from the Higgs boson decay in simulated signal events, before and after applying the regression. This technique improves the resolution on the di-jet invariant mass by about $15 \%$.

\section{Control regions and scale factors}

To check the compatibility between data and simulations, five control regions have been defined as follows:

- $Z+b$-jet : two b-tagged jets, zero leptons and Higgs mass veto between 100 and $140 \mathrm{GeV}$;

- $Z+$ light - jet : two no-b-tagged jets, zero leptons;

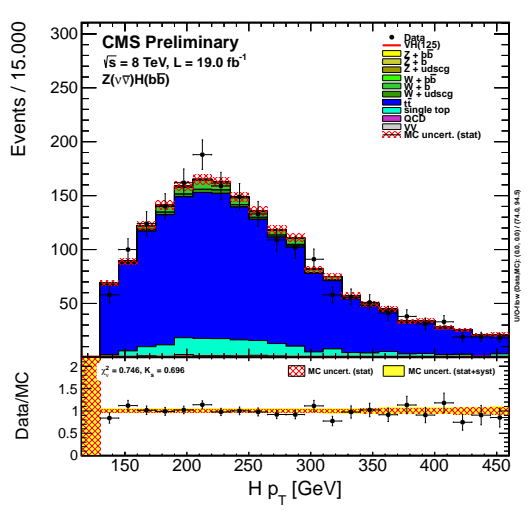

Figure 3. The Higgs candidate $p_{T}$ distribution for $t \bar{t}$ enriched samples. The di-jet momentum has been evaluated using the jet energy regression.

Table 1. $8 \mathrm{TeV}$ Data/MC scale factors for $W / Z+0 / 1 / 2 b$ jets and $t \bar{t}$. Errors include the statistical uncertainty from the fit, and a systematic uncertainty accounting for possible data/MC shape differences in the discriminating variables.

\begin{tabular}{cccc}
\hline \hline Background & $100<\mathrm{MET}(\mathrm{GeV})<130$ & $130<\mathrm{MET}(\mathrm{GeV})<170$ & $\mathrm{MET}(\mathrm{GeV})>170$ \\
\hline $\mathrm{W}+0 \mathrm{~b}$ & $0.83 \pm 0.02 \pm 0.04$ & $0.93 \pm 0.02 \pm 0.04$ & $0.93 \pm 0.02 \pm 0.03$ \\
$\mathrm{~W}+1 \mathrm{~b}$ & $2.30 \pm 0.21 \pm 0.11$ & $2.08 \pm 0.20 \pm 0.12$ & $2.12 \pm 0.22 \pm 0.10$ \\
$\mathrm{~W}+2 \mathrm{~b}$ & $0.85 \pm 0.24 \pm 0.14$ & $0.75 \pm 0.26 \pm 0.11$ & $0.71 \pm 0.25 \pm 0.15$ \\
$\mathrm{Z}+0 \mathrm{~b}$ & $1.24 \pm 0.03 \pm 0.09$ & $1.19 \pm 0.03 \pm 0.07$ & $1.17 \pm 0.02 \pm 0.08$ \\
$\mathrm{Z}+1 \mathrm{~b}$ & $2.06 \pm 0.06 \pm 0.09$ & $2.30 \pm 0.07 \pm 0.08$ & $2.13 \pm 0.05 \pm 0.07$ \\
$\mathrm{Z}+2 \mathrm{~b}$ & $1.25 \pm 0.05 \pm 0.11$ & $1.11 \pm 0.06 \pm 0.12$ & $1.12 \pm 0.04 \pm 0.10$ \\
$\mathrm{t} \mathrm{t}$ & $1.01 \pm 0.02 \pm 0.04$ & $0.99 \pm 0.02 \pm 0.03$ & $0.99 \pm 0.02 \pm 0.03$ \\
\hline \hline
\end{tabular}

- $W+b$-jet : two b-tagged jets, one lepton and Higgs mass veto between 100 and $140 \mathrm{GeV}$;

- $W+$ light - jet : two no-b-tagged jets, one lepton;

- $t \bar{t}$ : two b-tagged-jets, one lepton and possible additional jets.

Control plots have been produced for each region like that shown in Figure 3. A multi-fit of $W / Z+0 / 1 / 2$ b-jet and $t \bar{t}$ background scale factors have been performed, the results are show in Table 1. Scale factors differing from unity by less than $20 \%$ have been found for almost all backgrounds. $W / Z+1$ b-jet have a scale factors of about two. Similar discrepancies have also been found by other studies in CMS[8] and ATLAS[9].

\section{Signal extraction}

A signal pre-selection has been applied requiring two btagged jets and zero leptons. Additional cuts have been applied using three BDTs specialized to reduce $t \bar{t}, \mathrm{~W} / \mathrm{Z}+\mathrm{jets}$ and WW/WZ/ZZ backgrounds. A final BDT has then been used to separate the signal from all backgrounds. The main variables used to train the BDTs are:

- kinematics variables (e.g. Higgs jets $p_{T}$, Higgs jet $\Delta \eta$, $\Delta \phi($ jet, MET));

- b-tag discriminator of the two Higgs jets;

- features of additional jets (e.g. number, maximum b-tag discriminator, angular variables). 


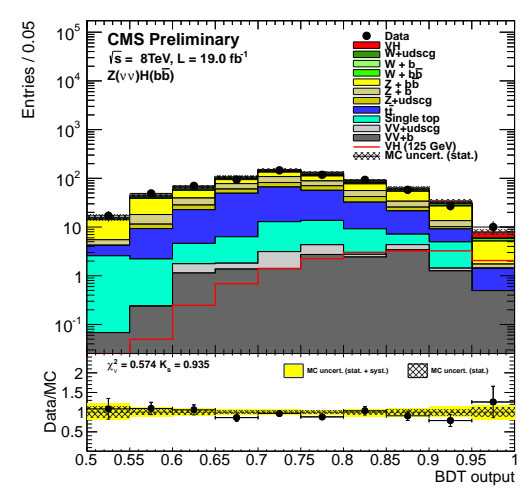

Figure 4. The invariant mass distributions after optimized cuts.

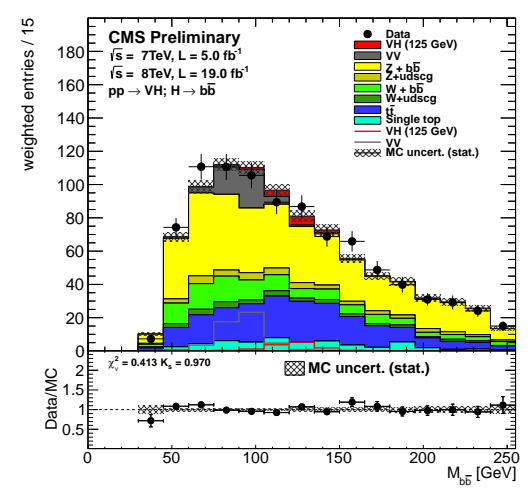

Figure 5. The final BDT distributions after all preselections, including the cuts on the three specialized BDTs, for data and simulations.

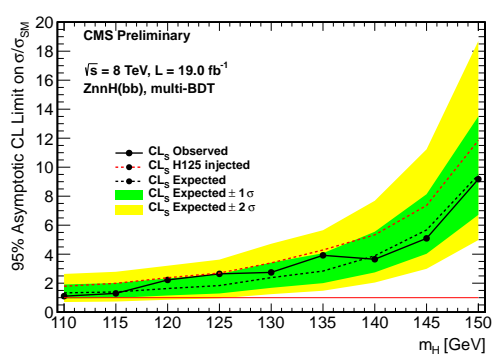

Figure 6. Exclusion plot of the SM Higgs boson cross section for $p p \rightarrow Z(v \bar{v}) H(b \bar{b})$ channel using the full $8 \mathrm{TeV}$ dataset. The plot shows the expected and observed $95 \%$ confidence level upper limits on the Higgs boson cross section times the $H \rightarrow b b$ branching ratio, with respect to the expectations from standard model Higgs. The limits are obtained using the full $8 \mathrm{TeV}$ dataset.

\section{Results}

To extract the final results, two shape analyses have been performed. The first one uses the multi-BDT approch de- scribed in the previus section. The second one uses the b-jet pair invariant mass distribution after optimized cuts. The distributions of the two variables are shown in Figure 4 and 5.

Figure 6 shows the exclusion plot obtained by fitting the output of the signal BDT for different Higgs boson mass hypotheses and using the confidence level (CL) method [10] to evaluate the lowest cross section excluded at 95\% CL. The plot shows an excess of about one standard deviation around $m_{H}=125 \mathrm{GeV}$. Here, an upper limit of 2.3 times SM Higgs cross section at $95 \%$ of confidence level has been observed, while an upper limit of 1.8 times the SM was expected in the hypothesis that the Higgs does not decay to $b \bar{b}$. The signal strength for $m_{H}=125 \mathrm{GeV}$, measured in units of the SM cross-section, is $1.0 \pm 0.8$.

\section{References}

[1] CMS Collaboration, "Observation of a new boson at a mass of $125 \mathrm{GeV}$ with the CMS experiment at the LHC”, Phys.Lett.B 716, 30-61 (2012).

[2] ATLAS Collaboration, "Observation of a new particle in the search for the Standard Model Higgs boson with the ATLAS detector at the LHC", Phys.Lett.B 716, 129 (2012).

[3] F. Englert and R. Brout, "Broken symmetry and the mass of gauge vector mesons", Phys. Rev. Lett. 13, 321 (1964).

[4] P. W. Higgs, "Broken symmetries, massless particles and gauge fields", Phys. Lett. 12, 132 (1964).

[5] CMS Collaboration, "The CMS experiment at the CERN LHC”, JINST 03, S08004 (2008).

[6] CMS Collaboration, "Search for the standard model Higgs boson produced in association with $\mathrm{W}$ or $\mathrm{Z}$ bosons, and decaying to bottom quarks (LHCP 2013)", CMS Physics Analysis Summary CMS-PAS-HIG-13012 (2013).

[7] CMS Collaboration Collaboration, "Identification of b-quark jets with the CMS experiment", JINST 8, P04013 (2013).

[8] CMS Collaboration, "Measurement of B hadron angular correlations in association to a $\mathrm{Z}$ boson", CMS Physics Analysis Summary CMS-PAS-EWK-11-015 (2012).

[9] ATLAS Collaboration, "Measurement of the crosssection for $\mathrm{W}$ boson production in association with $\mathrm{b}$ jets in pp collisions at $\mathrm{sqrt}(\mathrm{s})=7 \mathrm{TeV}$ with the ATLAS detector", arXiv:1302.2929.

[10] ATLAS and CMS Collaborations, LHC Higgs Combination Group, "Procedure for the LHC Higgs boson search combination in Summer 2011", ATL-PHYSPUB/CMS NOTE 2011-11, 2011/005 (2011). 